\title{
THE FREAK WAVE MYSTERY ??A NEW HYPOTHESIS FOR ITS OCCURRENCE
}

\author{
Nai-Kuang Liang \\ Institute of Oceanography, National Taiwan University, P.O. Box 23-13, Taipei, Taiwan, R.O.C., liangnk@ntu.edu.tw
}

Follow this and additional works at: https://jmstt.ntou.edu.tw/journal

Part of the Oceanography and Atmospheric Sciences and Meteorology Commons

\section{Recommended Citation}

Liang, Nai-Kuang (2007) "THE FREAK WAVE MYSTERY ??A NEW HYPOTHESIS FOR ITS OCCURRENCE," Journal of Marine Science and Technology. Vol. 15: Iss. 3, Article 9.

DOI: $10.51400 / 2709-6998.2397$

Available at: https://jmstt.ntou.edu.tw/journal/vol15/iss3/9

This Research Article is brought to you for free and open access by Journal of Marine Science and Technology. It has been accepted for inclusion in Journal of Marine Science and Technology by an authorized editor of Journal of Marine Science and Technology. 


\section{THE FREAK WAVE MYSTERY ??A NEW HYPOTHESIS FOR ITS OCCURRENCE}

Acknowledgements

The author thanks the Central Weather Bureau, Taiwan, Republic of China for the typhoon and wave data. Thanks are also extended to Dr. Tsai and Mr. Yeh for providing a picture and drawing a figure. 


\title{
THE FREAK WAVE MYSTERY - A NEW HYPOTHESIS FOR ITS OCCURRENCE
}

\author{
Nai-Kuang Liang*
}

Key words: freak wave, moving wind system, accumulation effect.

\begin{abstract}
Freak waves are known as a maritime myth, which have damaged large cargo and cruise ships. So far freak waves are explained by strong current focusing and nonlinear wave interaction. The latter may results in a single large wave. However this can still not explain the disappearance of the cargo ship Muenchen in December, 1978. This paper proposes a new hypothesis which stems from the wave/ swell energy flux accumulation due to the moving wind system. Nature might play the game by controlling the moving speed to approach the wave/swell group velocity.
\end{abstract}

\section{INTRODUCTION}

Freak waves, so-called rogue waves or monster waves are known as a maritime myth, because they are nearly impossible according to traditional ocean wave theory. State-of-the-art cargo ships and cruise ships that were heavily damaged by these waves disappeared. On January $1^{\text {st }} 1995$ an extreme single wave of 26 meters was measured under the Draupner oil-platform in the North Sea (http://www.math.uio.no/ karstent/ waves/index_en.html). On December 12, 1978 the cargo ship Muenchen, a state-of-the-art cargo ship, disappeared in the mid-Atlantic. In March, 2001, two reputable ships, designed to cope with the very worst conditions any ocean could throw at them, were crippled to the point of sinking. The Bremen and Caledonian Star were carrying hundreds of tourists across the South Atlantic. At 5am on 2 March the Caledonian Star's First Officer saw a $30 \mathrm{~m}$ wave bearing down on them (http:// www.bbc.co.uk/science/horizon/2002/freakwave. shtml).

The freak wave has been explained by the focusing current and nonlinear effects. However, the South Atlantic does not have strong currents. According to some research, it is completely feasible to have a freak

Paper Submitted 08/30/06, Accepted 11/15/06. Author for Correspondence: N.K. Liang. E-mail: liangnk@ntu.edu.tw.

*Institute of Oceanography, National Taiwan University, P.O. Box 23-13, Taipei, Taiwan, R.O.C. wave occur by natural, nonlinear processes from a random background of smaller waves. In such a case, it is hypothesized, an unusual, unstable wave type may form a single wave that 'sucks' energy from other waves, growing into a near-vertical monster itself, before becoming too unstable and collapsing shortly after. This is modeled by a wave equation known as the nonlinear Schroedinger equation, in which a normal and perfectly accountable wave begins to 'soak' energy from the waves immediately fore and aft. It is important to note that the spatio-temporal focusing in the Schroedinger equation can also occur when the nonlinearity is removed. In this case, focusing is primarily due to different waves coming into phase, rather than any energy transfer processes (http://en.wikipedia.org/wiki/ Freak_wave; [3], [5], [12]). However, a single nonlinear wave cannot make the large cargo ship like the Muenchen disappear. There must be another reason. So far the moving wind system has not been considered in freak wave theory. In the following a simple model will explain the connection between the moving wind system and the freak wave.

\section{THE ACCUMULATION EFFECT OF A WIND SYSTEM}

The typhoon or hurricane is a tropical atmospheric cyclone that may generate huge waves within its domain. The swell usually propagates faster than the typhoon itself and appears outside the typhoon. Young [13] stated also that SAR data from a number of hurricanes $[1,4,7,11]$ consistently showed swell ahead of hurricanes radiating out in a fan-shaped pattern from the center of the storm. The typhoon can be regarded as a wave generator. As the typhoon is stationary, the situation is different from that approaching a station. As the typhoon approaches the observer, the travel time for the wave energy becomes shorter. This results in an increase in the wave energy flux. This can be explained by the following: If Person A, who does not move, throws balls to Person $B$ at a fixed time interval and the speed of the ball relative to the ground is constant, then Person $\mathrm{B}$ receives balls at the same time interval. If Person A moves toward Person B, Person B receives balls at a 
shorter time interval. If the ball is like the energy, Person $B$ receives more energy flux from Person A, as Person A moves to Person B. Under the energy conservation assumption, the wave height modification factor used to multiply the swell height estimated from a stationary typhoon is as follows [9]:

$$
\lambda=\sqrt{T_{D} / T_{D}^{\prime}}
$$

in which $T_{D}$ is the swell appearance time period for a stationary typhoon, $T_{D}^{\prime}$ that for a moving typhoon. $T_{D}$ is the time lag for the successive typhoon data stream, usually 6 hours. $T_{D}^{\prime}$ is equal to $T_{D}-\Delta T_{\text {lag. }} . \Delta T_{\text {lag }}$ is the time difference between the swell propagation times for two successive typhoon data streams. The wave energy propagates in a wave group velocity, which is estimated to be 0.78 times the wave period $(\mathrm{m} / \mathrm{s})$ in linear wave theory. As the typhoon movement speed is close to the swell energy propagation speed, $T_{D}^{\prime}$ approaches zero. Following Eq. (1), the wave height modification factor $\lambda$ will be infinite. Of course it should not be infinite in reality but very large. In this paper it is assumed empirically that $\lambda$ has an upper limit 4 .

\section{CASE STUDY}

As a typhoon/hurricane approaches a location in the ocean quickly, the enhanced swell may be higher than those in the storm. If the typhoon approaching speed is close to the swell energy speed, i.e. the group velocity, a medium scale typhoon/hurricane may generate an extraordinary high swell. There were two serious accidents taking place in Taiwan. (1) At about 2 p.m., October 23, 1987, regardless of the invasion of Typhoon Lynn, 304 teachers and pupils of Hydraulic Elementary School of Pingtung County came to Mau-BiTou coast (near southern tip of Taiwan) for a tour. As the pupils walked one by one along aisle to the coral reef, suddenly huge waves attacked the coast and 9 pupils were drowned in the sea. The whole nation dropped in a great grief. At the meantime, the typhoon scale has transferred from "strong" to "medium" and its center was about $500 \mathrm{~km}$ away but has moved fast previously toward Mau-Bi-Tou. On the next day, the headline of United Daily News wrote: "Typhoon Far Away, Hazard Close to Eyes" [8]. (2) At about 4 a.m. of August 7, 1992, four fishing ships were totally destroyed by sudden huge waves in the vicinity of Suao Harbor. One man died, two persons were missing and five fishermen were wounded. As the accident was close to the harbor, some wrecks were drifted to shore. One fisherman reminded that he has never confronted such big waves in his 40 years' fishing career. Two days ago, a medium scale typhoon Janis had been in the area around $19^{\circ} \mathrm{N}, 136^{\circ} \mathrm{E}$ and moved fast toward Taiwan.

In 2003, two medium scale typhoons, i.e. Imbudo and Dujuan occurred near Taiwan. The typhoon tracks are shown in Figure 1. The typhoon data, provided by the Central Weather Bureau, are listed in Tables 1 and 2. The Central Weather Bureau has wave stations along the Eastern Coast of Taiwan. The Gueishandao and Chengkung wave stations are chosen for this study (Figure 1). The latitudes and longitudes for these two stations are Gueishandao $24.85^{\circ} \mathrm{N}, 121.93^{\circ} \mathrm{E}$; and Chengkung $23.13^{\circ} \mathrm{N}, 121.42^{\circ} \mathrm{E}$. Gueishandao is a small island. The hindcasted and measured typhoon waves at Gueishandao and Chengkung are presented in the following:

\section{Imbudo typhoon}

The hindcasted and measured wave heights are shown in Figure 2. Wave heights were also provided by the Central Weather Bureau (CWB) and a data buoy was operated by the Coastal Ocean Monitoring Center, National Cheng Kung University. The hindcasted data were higher than the measured data. During the Imbudo typhoon the South Bank Berth was severely damaged (Photo A). This berth was constructed by the Taiwan Tourism Bureau. Because the wave meter is located on the western side of the island, it is sheltered from southeastern swells. Because the data buoy recorded every 2 hours, high waves might be missed.

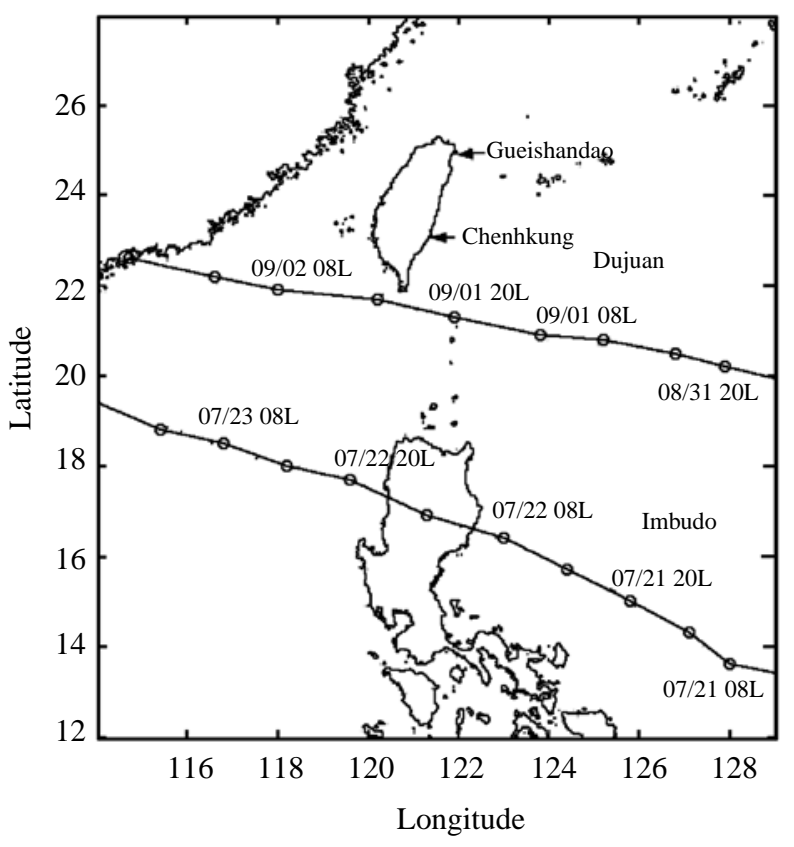

Fig. 1. Typhoon tracks and wave station locations. 
Table 1. Imbubo typhoon data

\begin{tabular}{cccccccc}
\hline Month & Date & $\begin{array}{c}\text { Time } \\
\text { (Local) }\end{array}$ & Lat. & Lon. & $\begin{array}{c}\text { Central pressure } \\
(\mathrm{hPa})\end{array}$ & $\begin{array}{c}\text { Max. wind } \\
(\mathrm{m} / \mathrm{s})\end{array}$ & $\begin{array}{c}\text { Speed radius } 7 \\
(\mathrm{~km})\end{array}$ \\
\hline 7 & 19 & 20 & 10.60 & 134.30 & 970 & 33 & 250 \\
7 & 20 & 02 & 10.80 & 133.40 & 965 & 35 & 250 \\
7 & 20 & 08 & 11.50 & 132.70 & 965 & 35 & 250 \\
7 & 20 & 14 & 12.10 & 131.60 & 955 & 40 & 300 \\
7 & 20 & 20 & 12.50 & 130.80 & 955 & 40 & 300 \\
7 & 21 & 02 & 13.30 & 129.60 & 955 & 40 & 300 \\
7 & 21 & 08 & 13.60 & 128.00 & 940 & 45 & 300 \\
7 & 21 & 14 & 14.30 & 127.10 & 940 & 45 & 300 \\
7 & 21 & 20 & 15.00 & 125.80 & 935 & 48 & 300 \\
7 & 22 & 02 & 15.70 & 124.40 & 940 & 45 & 300 \\
7 & 22 & 08 & 16.40 & 123.00 & 945 & 43 & 300 \\
7 & 22 & 14 & 16.90 & 121.30 & 955 & 40 & 300 \\
7 & 22 & 20 & 17.70 & 119.60 & 955 & 40 & 300 \\
7 & 23 & 02 & 18.00 & 118.20 & 955 & 40 & 300 \\
\hline
\end{tabular}

Table 2. Dujuan typhoon data

\begin{tabular}{cccccccc}
\hline Month & Date & $\begin{array}{c}\text { Time } \\
\text { (Local) }\end{array}$ & Lat. & Lon. & $\begin{array}{c}\text { Central pressure } \\
(\mathrm{hPa})\end{array}$ & $\begin{array}{c}\text { Max. wind } \\
(\mathrm{m} / \mathrm{s})\end{array}$ & $\begin{array}{c}\text { Speed radius 7 } \\
(\mathrm{km})\end{array}$ \\
\hline 8 & 31 & 02 & 18.50 & 132.20 & 970 & 33 & 250 \\
8 & 31 & 08 & 19.20 & 131.10 & 970 & 33 & 250 \\
8 & 31 & 14 & 19.80 & 129.60 & 965 & 35 & 250 \\
8 & 31 & 20 & 20.20 & 127.90 & 965 & 35 & 250 \\
9 & 01 & 02 & 20.50 & 126.80 & 965 & 35 & 250 \\
9 & 01 & 08 & 20.80 & 125.20 & 960 & 38 & 250 \\
9 & 01 & 14 & 20.90 & 123.80 & 950 & 43 & 250 \\
9 & 01 & 20 & 21.30 & 121.90 & 950 & 43 & 250 \\
9 & 02 & 02 & 21.70 & 120.20 & 950 & 43 & 250 \\
9 & 02 & 08 & 21.90 & 118.00 & 950 & 43 & 250 \\
9 & 02 & 14 & 22.20 & 116.60 & 955 & 40 & 250 \\
9 & 02 & 20 & 22.60 & 114.70 & 970 & 33 & 250 \\
\hline
\end{tabular}

Note: Radius 7 is the radius of beaufort scale No. 7 wind speed.

\section{Dujuan typhoon}

The hindcasted and measured wave heights at Gueishandao are shown in Figure 3. The hindcasted and measured data matches each other except at about 1 O'clock, Sept.2, when the wave height modification factor $\lambda$ was about 2 . Before and after that time the $\lambda$ was about 4 . Because the hindcasted swell arrival time has a time band, the middle datum can be those before and after. We can just skip the second datum. The hindcasted data is a little larger than the measured data but shows the same trend. As mentioned before, the wave station is somewhat sheltered from southeastern swells.

At Chengkung the hindcasted and measured wave heights, which are provided by the Central Weather Bureau, Rep. of China, are shown in Figure 4. During the measurement, the data were recorded continuously and quality-controlled. The hindcasted data matches the measured data well. The arrival time was 1 hour later than shown in the measured data. In Figure 4, the maximum wave height at Chengkung reached about $14 \mathrm{~m}$ at $0 \mathrm{~h}$, Sept. 2, 2003, of which the source, i.e. typhoon center, had been about $500 \mathrm{~km}$ away and the typhoon moving speed 16.3 knot. At 0h, Sept. 2, 2003, the typhoon Dujuan located just $188 \mathrm{~km}$ away southwestward to Chengkung. The typhoon movement speed was $17.5 \mathrm{knot}$ or $8.7 \mathrm{~m} / \mathrm{s}$. The maximum wind speed was $43 \mathrm{~m} / \mathrm{s}$ and the central pressure $950 \mathrm{hPa}$.

To estimate the maximum wave height inside a 


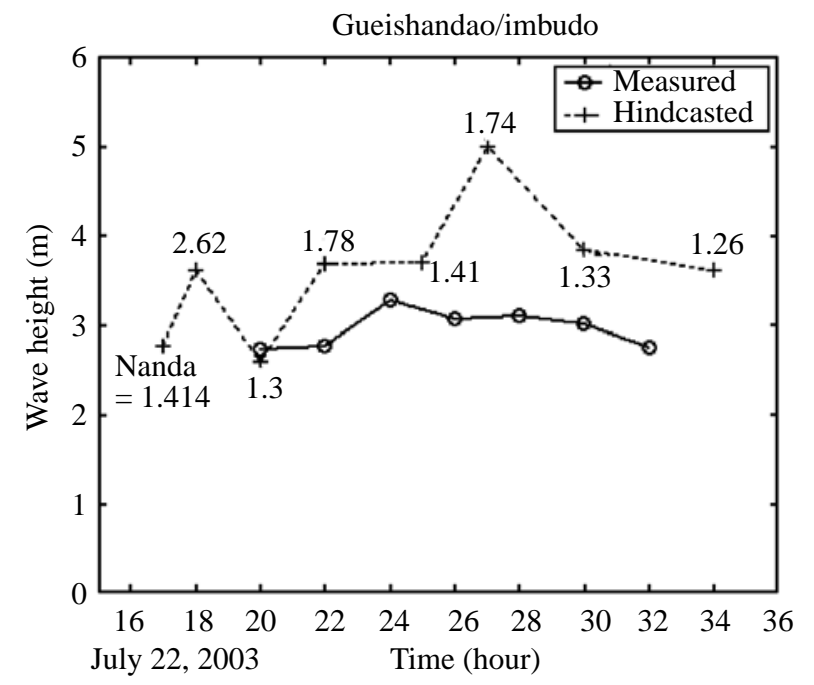

Fig. 2. Comparison of hindcasted and measured wave height at Gueishandoa during Imbudo typhoon (the numbers in the figure are the wave height modification factor $\lambda$ ).

typhoon, there are three well-known methods in the following. According to wave hindcast techniques [2, 13], the maximum significant wave height of typhoon/ hurricane is influenced mainly by the central pressure or the maximum wind speed, i.e. the strength of the typhoon/hurricane. The typhoon velocity of forward movement produces only a minor effect. Bretschneider and Tamaye proposed a formula to estimate the significant wave height $H_{R}$ at the maximum wind radius $R$ for a stationary hurricane as follows.

$$
H_{R}=K^{\prime} \sqrt{R \cdot \Delta P}
$$

in which $\Delta P$ is the central pressure reduction from normal in inches of mercury and $K^{\prime}$ a constant between 3.15-7.5. For a moving hurricane the significant wave height $H_{R V}$ becomes the following:

$$
H_{R V}=H_{R}\left(1+\frac{1}{2} \frac{V_{F} \cos \theta}{U_{R S}}\right)^{2}
$$

in which $V_{F}$ is the hurricane forward velocity, $U_{R S}$ the 10 -minute average wind speed at the 10 -meter reference level at $R$ and $\theta$ the angle between $V_{F}$ and $U_{R S}$. The limitation of Eq. (3) is that $V_{F} \leq V_{C}$, where $V_{C}$ is the critical forward speed. Similarly, as in Figure 2 of Young's paper, the maximum significant wave height in hurricane increases drastically with increasing maximum wind speed $V_{\max }$ and increases gradually with increasing forward speed $V_{F}$ until a critical one. Otherwise, Hsu, S.A. doesn't consider the hurricane moving speed [6]. The maximum wave height in meters

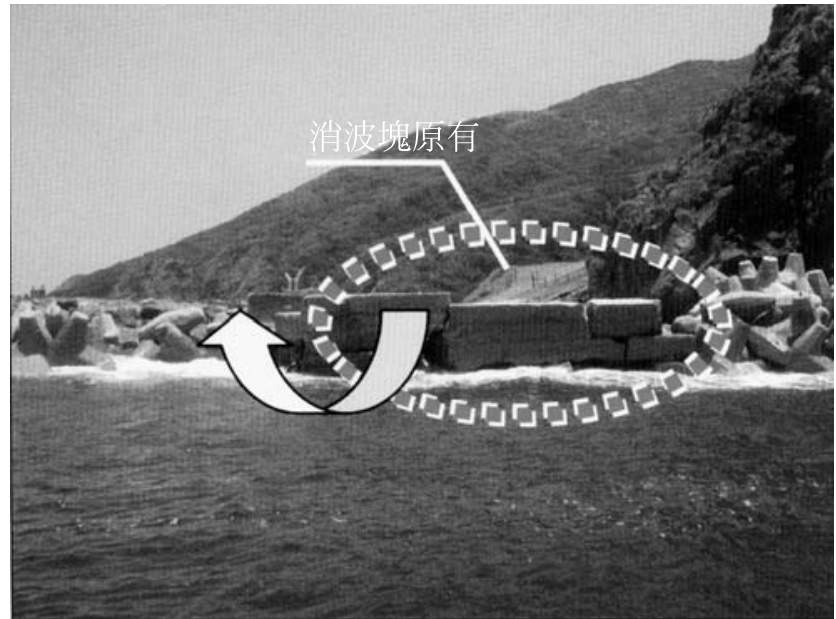

Photo A The armored blocks suspended and moved to the berth by swell at Gueishandao (provided by Dr. Peter Y. Tsai)

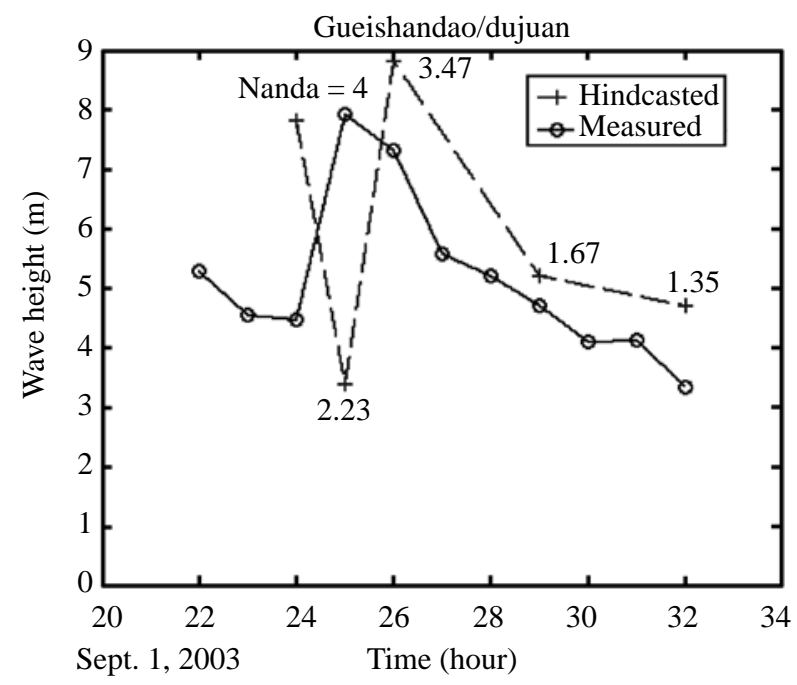

Fig. 3 Comparison of hindcasted and measured wave height at Gueishandoa during Dujuan typhoon (the numbers in the figure are the wave height modification factor $\lambda$ ).

$H_{s}$ is as follows:

$$
H_{s}=0.2\left(1013-P_{c}\right)
$$

where $P_{c}$ is the typhoon central pressure in hPa.

Using Eqs. (2) and (3), the maximum wave height within the Dujuan typhoon at 0h, Sept. 2, 2003, considering the movement velocity, was estimated at $11.2 \mathrm{~m}$. Employing Figure 3 of the reference [13], the maximum wave height within the Dujuan typhoon, considering the movement velocity, was estimated at about 10-11 m. By Eq. (4), the maximum wave height amounted to $12.6 \mathrm{~m}$, which was still less than the measured wave height 14 meters at Chengkung. 


\section{DISCUSSION AND CONCLUSION}

The breakwater at Gueishandao was destroyed by the wave swell while the Imbudo typhoon was $1200 \mathrm{~km}$ away, this asserts that a distant typhoon/hurricane may generate very high swell height as the typhoon/hurricane approaches quickly to any location in the ocean. The berth was further damaged by the following Dujuan typhoon.

Because the wave period of a medium scale typhoon/hurricane, of which the central pressure is around $960 \mathrm{hPa}$, is not very large, the probability of a larger accumulation/Doppler effect is higher than that for a strong typhoon/hurricane. For a strong Doppler effect, i.e. larger $\lambda$ value, the wave appearance time becomes so short that the data may probably be missed.

At 0h, Sept. 2, 2003 the wave height was measured at nearly $14 \mathrm{~m}$ at Chengkung which was generated from the Typhoon Dujuan, while it was about $500 \mathrm{~km}$ away and its movement speed about 16.3 knots. At the same time, i.e. 0h, Sept. 2, the maximum wave height inside the Typhoon Dujuan was estimated to be only about 12 $\mathrm{m}$. The maximum wave height measured at Gueishandao, located farther away to the north, amounted to 8 meters in one hour later.

The above-mentioned wave height is the so called "significant wave height", which is the average of the highest $1 / 3$ waves in a period wave record, say 20 minutes. According to Rayleigh distribution [10], the maximum wave height in the record is 1.27 times $14 \mathrm{~m}$, i.e. $18 \mathrm{~m}$, or more. Other than ty-phoons or hurricanes, there are other wind systems, such as the extra-tropical

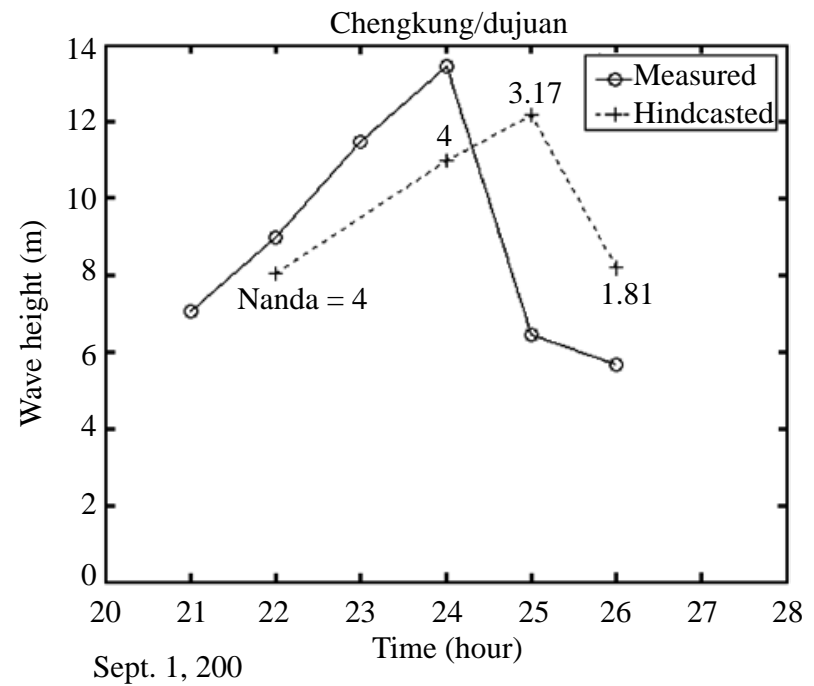

Fig. 4. Comparison of hindcasted and measured wave height at Chengkung during Dujuan typhoon (the numbers in the figure are the wave height modification factor $\lambda$ ). cyclone, which is active at the mid-latitude in both the northern and southern hemispheres and always moves westerly. All of these wind systems may have the same possibilities.

The accumulation effect of a moving wind system is significant while the swell appearance time period $T_{D}^{\prime}$ is short. The shorter the time period the higher the wave. The accumulated huge wave/swell appears suddenly. This agrees with the word "freak" and may uncover the mystery. The mechanism is controlled by the wind system movement speed. Nature might play the Chinese Kung-Fu to concentrate the wave/swell energy within a very short time.

\section{ACKNOWLEDGEMENT}

The author thanks the Central Weather Bureau, Taiwan, Republic of China for the typhoon and wave data. Thanks are also extended to Dr. Tsai and Mr. Yeh for providing a picture and drawing a figure.

\section{REFERENCE}

1. Beal, R.C., Gerling, T.W., Irvine, D.E., Monaldo, F.M., and Tilley, D.G., "Spatial Variations of Ocean Wave Directional Spectra from the SEASAT Synthetic Aperture Radar," Journal of Geophysics Research, Vol. 91 (C2), pp. 2433-2449 (1986).

2. Bretschneider, C.L. and Tamaye, E.E., "Hurricane Wind and Wave Forecasting Techniques," Proceedings $15^{\text {th }}$ International Coastal Engineering Conference, pp. 202237 (1976).

3. Dole, J.W. and Peregrine, D.H., "Wave-Wave Modulation," International Conference on Coastal Engineering, ASCE, pp. 163-175 (1986).

4. Gonzalez, F.I., "SEASAT Wind and Wave Observations of Northeast Pacific Hurricane Iva, August 13, 1978," Journal of Geophysics Research, Vol. 78(C5), pp. 34313438 (1982).

5. Henderson, K.L., Peregrine, D.H., and Dole, J.W., "Unsteady Water Wave Modulation: Fully Nonlinear Solutions and Comparison with the Nonlinear Schroedinger Equation," Wave Motion, Vol. 29, pp. 341-361 (1999).

6. Hsu, S.A., "Estimating Hurricane Waves," Mariners Weather Log, Vol. 38, No. 1, p. 68 (1994).

7. King, D.B. and Shemdin, O.H., "Radar Observations of Hurricane Wave Directions," Proceedings of the $16^{\text {th }}$ International Conference on Coastal Engineering, Hamburg, West Germany, pp. 209-226 (1978).

8. Liang, N.K., "A Study on Typhoon Swell Height Prediction," Acta Oceanographica Taiwanica, No. 25, pp. 77-86 (1990).

9. Liang, N.K., "The Typhoon Swell Doppler Effect," Ocean Engineering, Vol. 30, pp. 1107-1115 (2003). 
10. Longuet-Higgins, M.S., "On the Statistical Distribution of the Heights of Sea Waves," Journal of Marine Research, Vol. 11, pp. 245-266 (1952).

11. McLeish, W. and Ross, D.B., "Imaging Radar Observations of Directional Properties of Ocean Waves," Journal of Geophysics Research, Vol. 88(C7), pp. 44074419 (1983).
12. Peregrine, D.H., "Water Wave and Their Development in Space and Time," Proceedings of the Royal Society, London, Vol. A400, pp. 1-18 (1985).

13. Young, I.R., "Parametric Hurricane Wave Prediction Model," Journal of Waterway, Port, Coastal and Ocean Engineering, Vol. 114, No. 5, pp. 637-652 (1987). 